\title{
Eliminating Tromboning Mobile Call Setup for International Roaming Users
}

\author{
Yi-Bing Lin, Fellow, IEEE
}

\begin{abstract}
Existing mobile service providers allow their customers to receive telecommunications services when they roam to other countries. However, in the standard mobile call setup procedure, a call from the visited country to a roaming customer at that country results in two international calls. This phenomenon is referred to as the tromboning effect. In this paper, we propose a third-party solution that resolves the tromboning problem by replacing two international calls with two local calls. Besides huge saving in voice/data path, analytic modeling and simulation experiments indicate that our solution is effective in call setup signaling. Also, our solution does not need to modify the existing mobile telecommunications systems.
\end{abstract}

Index Terms-International roaming, mobile telecommunications service, mobility management, tromboning, voice over IP (VoIP).

\section{INTRODUCTION}

G LOBAL roaming of mobile telecommunications services is widely available today, where the mobile service providers offer their customers to receive telecommunications services when they travel to other countries. However, international roaming is very expensive either for voice or data integrated services [1]. Consider the following scenario [2]: Jenny in Taiwan travels to the US. Her friend John in the US calls her. Since both John and Jenny are in the US, they anticipate that this call is "local". Unfortunately, the standard roaming call setup procedure [3] results in two international calls as illustrated in the following steps (see Figure 1):

Step A.1: John dials Jenny's mobile phone number +886931111111. Through the SS7 Initial Address Message (IAM), the call is set up to Jenny's Gateway Mobile Switching Center (GMSC) in Taiwan through the US and the Taiwan International Switching Centers (ISCs); see path $($ a $) \rightarrow($ b $) \rightarrow($ c $) \rightarrow(d) \rightarrow($ e) in Figure 1 .

Step A.2: The GMSC queries the Home Location Register (HLR; Figure 1 (f)) to identify the Mobile Station Roaming Number (MSRN) of the target MSC (Figure 1 (h)) visited by Jenny's mobile station (MS; i.e., her mobile handset at Figure 1 (i)). Specifically, the HLR communicates with the Visitor Location Register (VLR) of the target MSC (Figure $1(\mathrm{~g})$ ) to obtain the MSRN. The signaling path is $(\mathrm{f}) \rightarrow(\mathrm{d}) \rightarrow(\mathrm{c}) \rightarrow(\mathrm{g}) \rightarrow(\mathrm{c}) \rightarrow(\mathrm{d}) \rightarrow$ $(\mathrm{f}) \rightarrow(\mathrm{e})$.

Manuscript received December 4, 2007; revised February 20, 2008; accepted February 25, 2008. The associate editor coordinating the review of this paper and approving it for publication was Y. Fang.

Y.-B. Lin is with the Department of Computer Science, National Chiao Tung University, Hsinchu 30010, Taiwan, R.O.C. (e-mail: liny@csie.nctu.edu.tw). Y.-B. Lin is also with the Institute of Information Science, Academia Sinica, Nankang, Taipei, Taiwan.

Digital Object Identifier 10.1109/T-WC.2009.071360

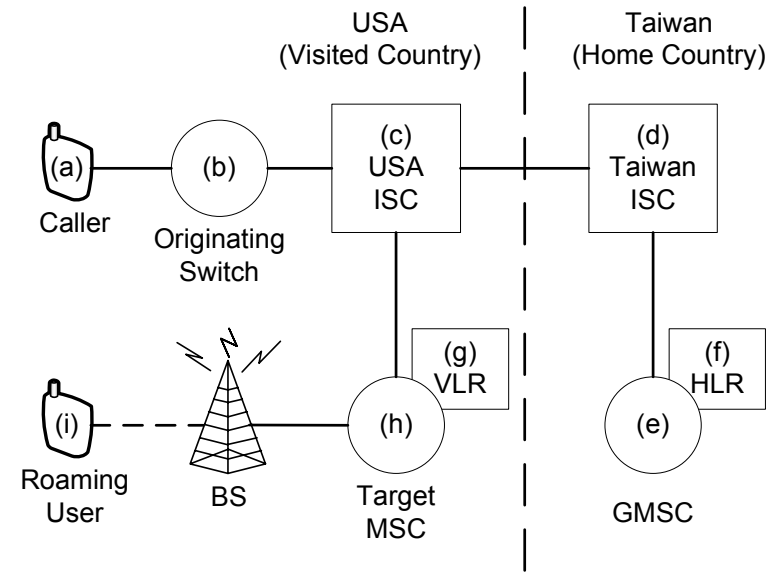

Fig. 1. International call setup in existing mobile telecommunications networks.

Step A.3: According to the MSRN (which indicates the address of the target MSC), the GMSC sets up the call to Jenny through the visited US mobile network; see path $(\mathrm{e}) \rightarrow(\mathrm{d}) \rightarrow(\mathrm{c}) \rightarrow(\mathrm{h}) \rightarrow(\mathrm{i})$.

Step A.4: When Jenny's MS rings, an SS7 Address Complete Message (ACM) is sent from the target MSC to the originating switch through path $(\mathrm{h}) \rightarrow(\mathrm{c}) \rightarrow$ (d) $\rightarrow$ $($ e) $\rightarrow$ (d) $\rightarrow$ (c) $\rightarrow$ (b). When Jenny picks up the phone, the call is connected.

At the end of Step A.4, John and Jenny start conversation through path (a)-(b)-(c)-(d)-(e)-(d)-(c)-(h)-(i). The above procedure results in two international connections for the call; where John pays for the international call with path (a)-(b)(c)-(d)-(e), and Jenny pays for the international call with path (e)-(d)-(c)-(h)-(i).

Clearly, the above "tromboning" call path consumes extra network resources, and the call parties need to pay more costs. This problem was seldom studied in the literature. In [4], we proposed several solutions to eliminate the tromboning effect at the cost of modifying the existing mobile telecommunications network nodes. In this paper, we propose a third-party solution that utilizes Internet to resolve the tromboning problem by replacing two international calls with two local calls. Our solution does not need to modify the existing mobile telecommunications systems. Therefore, no extra roaming agreements need to be signed among the mobile operators of different countries. 


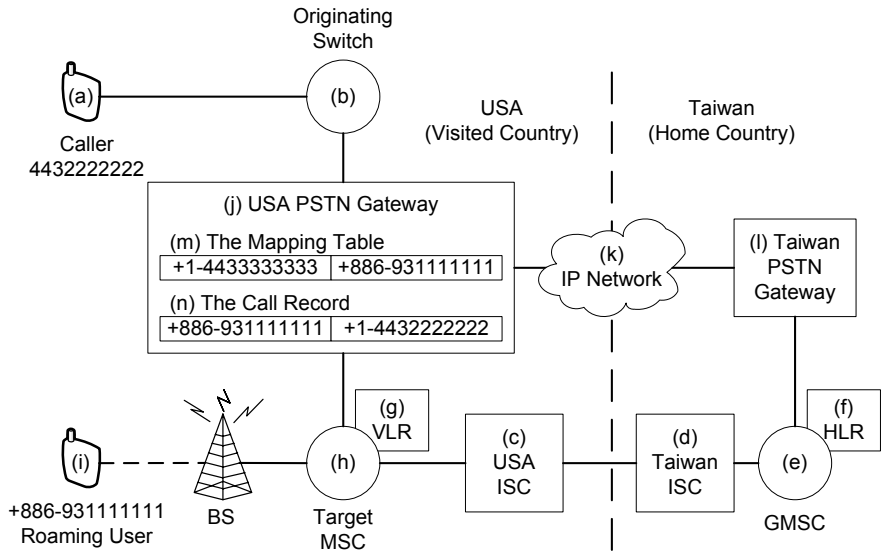

Fig. 2. International call setup in our approach.

\section{A New Approach to Eliminate the tromboning EFFECT}

A business person typically has a local business phone number in each of the countries she frequently visits. Therefore, in our solution, a mobile customer is assigned a local phone number (typically a fixed network number) for each of the countries she frequently visits. Suppose that the solution provider of our approach is from Taiwan. A Taiwan subscriber Jenny has the mobile phone number +886-931111111, a Taiwan phone number $+886-5722222$, and a US phone number $+1-4433333333$. These local phone numbers are assigned by the solution provider (to be elaborated in Issue 2 of Section V). The solution provider deploys a PSTN gateway [5] in each country (Figure $2(\mathrm{j})$ ) of its service coverage. Every PSTN gateway is connected to the PSTN (see links (j)-(b), (j)-(h), and (l)-(e) in Figure 2) and to a telecom-grade IP Network (Figure $2(\mathrm{k})$ ).

A PSTN gateway maintains a table that maps the local phone number (at the country where the PSTN gateway resides) to the mobile phone number of each subscriber. For example, the PSTN gateway in the US maps Jenny's US local phone number $+1-4433333333$ to her mobile phone number $+886-931111111$ (Figure $2(\mathrm{~m})$ ). The PSTN gateway also maintains a call record table. When John at the US (with phone number $+1-4432222222$ ) calls Jenny, the PSTN gateway creates a call record that records the called mobile phone number and the calling phone number pair $(+886-$ 931111111,+1-4432222222); see Figure 2 (n).

Suppose that Jenny travels from Taiwan to Baltimore, USA. When she arrives at the Baltimore Washington International Airport, she turns on her MS, and switches the phone to the international roaming mode by entering the roaming place as USA (to be elaborated in Issue 3 of Section V). We note that Jenny's MS follows the normal mobile location update procedure for international roaming [2]. When John in the US calls Jenny, the call setup procedure works as follows:

Step B.1: John dials Jenny's US phone number 4433333333. The originating switch (Figure 2 (b)) routes the call to the local PSTN gateway; see path (a) $\rightarrow$ (b) $\rightarrow$ (j) in Figure 2 .

Step B.2: The US PSTN gateway uses the number 4433333333 to retrieve Jenny's mobile phone number
+886-931111111 in the mapping table (see Figure 2 (m)). Based on this mobile phone number, the US PSTN gateway sets up the call to Jenny's GMSC (Figure 2 (e)) through the VoIP network (Figure $2(\mathrm{k})$ ) and Taiwan's PSTN gateway (Figure $2(1)$ ). These steps are the same as those for standard skype-out routing; see path (j) $\rightarrow$ $(\mathrm{k}) \rightarrow(\mathrm{l}) \rightarrow(\mathrm{e})$ in Figure 2.

Step B.3: Following the standard mobile phone call set up procedure (i.e., Steps A.2 and A.3), the GMSC forwards the IAM message to set up the call to Jenny's MS through the visited mobile network; see Figure 2, path (f) $\rightarrow(\mathrm{d}) \rightarrow$ $(\mathrm{c}) \rightarrow(\mathrm{g}) \rightarrow(\mathrm{c}) \rightarrow(\mathrm{d}) \rightarrow$ (f) $\rightarrow$ (e) for retrieving the MSRN, and path (e) $\rightarrow$ (d) $\rightarrow$ (c) $\rightarrow$ (h) $\rightarrow$ (i) for call setup.

Step B.4: When Jenny's MS is paged by the base station (BS) of the target MSC, the MS first checks if the international mode is active, and if the caller ID is from the visited country (i.e., the US in our example). If so, Steps B.4 (a), (b), and B.5 are executed. Otherwise, the MS skips these steps, and follows the standard mobile call setup procedure (Step A.4).

Step B.4 (a): Jenny's MS detects the international roaming mode, and automatically rejects the mobile call setup by sending the ACM message with call reject [2]; the message is delivered through path (i) $\rightarrow$ (h) $\rightarrow$ $($ c) $\rightarrow$ (d) $\rightarrow$ (e) in Figure 2. The GMSC forwards the $A C M$ (reject) message to inform the US PSTN gateway that the call is canceled through path $(\mathrm{e}) \rightarrow(\mathrm{l}) \rightarrow(\mathrm{k}) \rightarrow$ (j) in Figure 2.

Step B.4 (b): In parallel with Step B.4 (a), Jenny's MS rings. From the caller ID (i.e., +1-4432222222) carried by the IAM message at Step B.3 (issued from John's side), Jenny determines if she wants to answer the call. If so, the MS retrieves the address of the US PSTN gateway and directly sets up the call to the gateway (through a new IAM message issued from Jenny's side); see path (i) $\rightarrow(\mathrm{h}) \rightarrow(\mathrm{j})$ in Figure 2 .

Step B.5: One of the following two cases may occur in the US PSTN gateway after Step B.2:

Case I: The ACM message from the GMSC (Step B.4 (a)) arrives at the US PSTN gateway first. The PSTN gateway sets a call-back timer. There are two possibilities:

Case I (a): If a call setup request from Jenny's MS (i.e., the IAM message sent from the target MSC at Step B.4 (b)) arrives at the US PSTN gateway before the call-back timer expires, the gateway uses the caller ID (i.e., Jenny's mobile phone number +886-931111111) to retrieve John's phone number from the call record table in Figure 2 (n); note that this call record was created at Step B.2. The PSTN gateway then bridges the call (path (a)-(b)-(j)-(h)(i) in Figure 2), and both John and Jenny can start conversation.

Case I (b): If the call-back timer expires, then the US PSTN gateway clears the call record in the table, and rejects the call setup request issued from John at Step B.1. If the call-back request from Jenny (Step B.4 (b)) arrives after the call-back timer has 
expired, then the PSTN gateway rejects Jenny's call-back request. In this case, the call is lost, and Jenny may call back again by directly dialing John's phone number (using the caller ID provided in the IAM message at Step B.3).

Case II: Jenny's call-back request (Step B.4 (b)) arrives at the PSTN gateway before call release from the GMSC (Step B.4 (a)). The US PSTN gateway bridges the call as in Case I (a). The call release from the GMSC (the ACM message delivered at Step B.4 (a)) is not forwarded to the originating switch.

In the above procedure, the call from John to Jenny consists of two local phone calls (paths (a)-(b)-(j) and (i)-(h)-(j)). Therefore, the usage of network resources are significantly reduced as compared with the standard international (tromboning) mobile call procedure described in Steps A.1-A.4. In terms of call setup signaling, the proposed approach needs an extra message delivery through path (i)-(h)-(j). However, this local message delivery is conducted in parallel with the international ACM message delivery, and therefore the cost is minor.

\section{Analytic Modeling for Call Setup Signaling}

From Section II, it is clear that our approach significantly reduces the cost of international calls for roaming users. On the other hand, one may suspect the effectiveness of our call setup procedure. We utilize two performance measures to investigate call setup signaling for our approach:

- $p_{1}$ : the probability that Case I (b) occurs in our approach; a small $p_{1}$ means that the call is unlikely to drop.

- $p_{2}$ : the probability that the call setup time for our approach is longer than the standard international mobile call setup; a small $p_{2}$ means that our approach can set up an international call faster than the existing approach.

In the following two subsections, we analytically derive the probabilities $p_{1}$ and $p_{2}$.

\section{A. Derivation for $p_{1}$}

In Figure 2, let $t_{1}$ be the ACM message delay for path $(\mathrm{i}) \rightarrow(\mathrm{h}) \rightarrow(\mathrm{c}) \rightarrow(\mathrm{d}) \rightarrow(\mathrm{e}) \rightarrow(\mathrm{l}) \rightarrow(\mathrm{k}) \rightarrow(\mathrm{j})$. Let $t_{2}$ be the IAM message delay for path $(\mathrm{i}) \rightarrow(\mathrm{h}) \rightarrow(\mathrm{j})$. Let constant $T$ be the timeout period of the call-back timer in the US PSTN gateway. Then $p_{1}$ is the probability that $t_{2}>t_{1}+T$. We assume that $t_{1}$ is a Gamma random variable with the density function $f_{1}\left(t_{1}\right)=\left[\frac{\beta\left(\beta t_{1}\right)^{\alpha-1}}{\Gamma(\alpha)}\right] e^{-\beta t_{1}}$ (where $\Gamma(\cdot)$ is the Gamma function, and $\alpha$ is a non-negative real number), the mean value $E\left[t_{1}\right]=\frac{\alpha}{\beta}$, and the variance $V_{1}=\frac{\alpha}{\beta^{2}}$. The Laplace transform for the $t_{1}$ random variable is

$$
\begin{gathered}
f_{1}^{*}(s)=\left(\frac{\beta}{\beta+s}\right)^{\alpha}, \quad \text { and } \\
\frac{d^{m} f_{1}^{*}(s)}{d s^{m}}=\left[\frac{(\alpha+m-1) !}{(\alpha-1) !}\right]\left[\frac{(-1)^{m} \beta^{\alpha}}{(\beta+s)^{\alpha+m}}\right]
\end{gathered}
$$

Let $t_{2}$ be an Erlang random variable with the density function

$$
f_{2}\left(t_{2}\right)=\left[\frac{\lambda\left(\lambda t_{2}\right)^{k-1}}{(k-1) !}\right] e^{-\lambda t_{2}}
$$

(where $k$ is a non-negative integer) with the mean value $E\left[t_{2}\right]=\frac{k}{\lambda}$, the variance $V_{2}=\frac{k}{\lambda^{2}}$, and the distribution function

$$
F_{2}\left(t_{2}\right)=1-\sum_{n=0}^{k-1}\left[\frac{\left(\lambda t_{2}\right)^{n}}{n !}\right] e^{-\lambda t_{2}}
$$

We assume $t_{2}$ to be Erlang distributed, which is often used in telecommunications modeling [6], [7], [8], [9]. Because more network nodes are visited in the international ACM signaling path with delay $t_{1}$ than the local IAM signaling path with delay $t_{2}$, the variation of $t_{1}$ is more significant than that for $t_{2}$. Therefore, we assume $t_{1}$ to be Gamma distributed (note that the Gamma distribution is a generalization of the Erlang distribution). The Gamma distribution is also widely used in telecommunications modeling [10], [11], [12] because it has been shown that the distribution of any positive random variable can be approximated by a mixture of Gamma distributions (see Lemma 3.9 in [13]). We can measure the signaling delays from the field operations and then generate the Gamma/Erlang distributions from the measured data.

From (2) and (3), we have

$$
\begin{aligned}
p_{1} & =\operatorname{Pr}\left[t_{2}>t_{1}+T\right] \\
& =\int_{t_{1}=0}^{\infty} \int_{t_{2}=t_{1}+T}^{\infty}\left[\frac{\lambda\left(\lambda t_{2}\right)^{k-1}}{(k-1) !}\right] e^{-\lambda t_{2}} f_{1}\left(t_{1}\right) d t_{2} d t_{1} \\
& =\sum_{n=0}^{k-1}\left[\left(\frac{\lambda^{n}}{n !}\right) e^{-\lambda T}\right] \int_{t_{1}=0}^{\infty}\left(t_{1}+T\right)^{n} f_{1}\left(t_{1}\right) e^{-\lambda t_{1}} d t_{1} \\
& =\sum_{n=0}^{k-1} \sum_{m=0}^{n}\left[\left(\frac{\lambda^{n}}{n !}\right)\left(\begin{array}{c}
n \\
m
\end{array}\right) T^{n-m} e^{-\lambda T}\right] \\
& \times \int_{t_{1}=0}^{\infty} t_{1}^{m} f_{1}\left(t_{1}\right) e^{-\lambda t_{1}} d t_{1} \\
= & \left.\sum_{n=0}^{k-1} \sum_{m=0}^{n}\left[\frac{(-1)^{m}(\lambda T)^{n}}{m !(n-m) ! T^{m}}\right] e^{-\lambda T}\left[\frac{d^{m} f_{1}^{*}(s)}{d s^{m}}\right]\right|_{s=\lambda}(4)
\end{aligned}
$$

If $k=1$, then $p_{1}=e^{-\lambda T} f_{1}^{*}(\lambda)$. If $f_{1}\left(t_{1}\right)$ is a Gamma density function, then from (1), (4) is re-written as

$$
p_{1}=\sum_{n=0}^{k-1} \sum_{m=0}^{n}\left[\frac{(\alpha+m-1) !}{(\alpha-1) ! m !(n-m) !}\right]\left[\frac{\lambda^{n} T^{n-m} \beta^{\alpha}}{(\beta+\lambda)^{\alpha+m}}\right] e^{-\lambda T}
$$

In (4), probability $p_{1}$ is exponentially improved by the callback timeout period $T$ through the term $e^{-\lambda T}$.

\section{B. Derivation for $p_{2}$}

The signaling path for the international tromboning call setup can be partitioned into the following segments (see Figure 1):

Segment A.1: $($ a $) \rightarrow$ (b) $\rightarrow$ (c) (the call request from the caller and the IAM signaling from the originating switch to the US ISC), (c) $\rightarrow$ (d) $\rightarrow$ (e) $\rightarrow$ (f) $\rightarrow$ (d) $\rightarrow$ (c) $\rightarrow$ (g) $\rightarrow$ $(\mathrm{c}) \rightarrow(\mathrm{d}) \rightarrow(\mathrm{f}) \rightarrow(\mathrm{e}) \rightarrow$ (d) $\rightarrow$ (c) (the tromboning IAM signaling and the MSRN query), and $(\mathrm{c}) \rightarrow(\mathrm{h}) \rightarrow$ (i) (the IAM signaling from the US ISC to the target MSC/called party).

Segment A.2: (i) $\rightarrow$ (h) $\rightarrow$ (c) $\rightarrow$ (d) $\rightarrow$ (e) $\rightarrow$ (d) $\rightarrow$ (c) (the tromboning ACM signaling from the target $\mathrm{MSC} / \mathrm{called}$ party to the US ISC). 
Segment A.3: (c) $\rightarrow$ (b) $\rightarrow$ (a) (the ACM signaling from the US ISC to the originating switch/calling party).

The signaling path for our solution is partitioned into the following segments (see Figure 2):

Segment B.1: (a) $\rightarrow$ (b) $\rightarrow$ (j), (the call request from the caller and the IAM signaling from the originating switch to the US PSTN gateway), (j) $\rightarrow(\mathrm{k}) \rightarrow(\mathrm{l}) \rightarrow(\mathrm{e}) \rightarrow(\mathrm{f}) \rightarrow$ $(\mathrm{d}) \rightarrow(\mathrm{c}) \rightarrow(\mathrm{g}) \rightarrow(\mathrm{c}) \rightarrow(\mathrm{d}) \rightarrow(\mathrm{f}) \rightarrow(\mathrm{e}) \rightarrow(\mathrm{d}) \rightarrow(\mathrm{c})$ (the tromboning IAM signaling from the US PSTN gateway to the US ISC and the MSRN query), and (c) $\rightarrow(\mathrm{h}) \rightarrow$ (i) (the IAM signaling from the US ISC to the target MSC/called party)

Segment B.2: $(\mathrm{i}) \rightarrow(\mathrm{h}) \rightarrow$ (j) (the IAM signaling from the called party/target MSC to the US PSTN gateway).

Segment B.3 (a): (j) $\rightarrow$ (b) $\rightarrow$ (a) (the ACM signaling from the US PSTN gateway to the originating switch/calling party).

Segment B.3 (b): $($ j) $\rightarrow$ (h) $\rightarrow$ (i) (the ACM signaling from the US PSTN gateway to the target MSC/called party).

Note that the signaling for canceling the mobile call setup (Step B.4 (a)) and the call-back timeout $T$ do not need to be considered in the above call setup path. We make the following assumptions:

- The message delay for Segment A.1 is the same as that for Segment B.1 (which are international tromboning signaling in both scenarios). For simplicity, we exclude these segments in deriving $p_{2}$.

- The delay for Segment A.2 is assumed to have the same distribution as that for $t_{1}$ in Section III-A.

- The delay for Segment B.2 is assumed to have the same distribution as that for $t_{2}$ in Section III-A.

- The delay $t_{3}$ for Segment A.3 and the delay $t_{4}$ for Segment B.3 (a) are identical random variables (which are local call signaling to the calling party).

- The delay $t_{5}$ for Segment B.3 (b) is identical to $t_{4}$ (which are local call signaling to the called and the calling parties, respectively).

For other distributions of the above transmission delays, similar results are observed and will not be presented here. The call setup signaling delay for our approach is longer than the existing (traditional tromboning) approach with the probability $p_{2}=\operatorname{Pr}\left[t_{2}+\max \left(t_{4}, t_{5}\right)>t_{1}+t_{3}\right]$.

We assume that $t_{1}$ has an arbitrary distribution. Delays $t_{2}$, $t_{3}, t_{4}$, and $t_{5}$ are assumed to be Exponential random variables with the density functions $f_{2}(t)=f_{3}(t)=f_{4}(t)=f_{5}(t)=$ $\lambda e^{-\lambda t}$. Although the Exponential assumption may not actually reflect the distributions for the signaling delays, it does provide the mean value analysis [14] for a primary study on the trends of the signaling delay impact. Also, analytic results based on Exponential assumption can be used to validate the simulation experiments. Note that this assumption can be easily relaxed in the validated simulation experiments.

$$
\begin{aligned}
\text { Let } t_{6} & =\max \left(t_{4}, t_{5}\right), \text { then } \\
f_{6}\left(t_{6}\right) & =\int_{t_{5}=0}^{t_{6}} \lambda e^{-\lambda t_{5}} \lambda e^{-\lambda t_{6}} d t_{5}+\int_{t_{4}=0}^{t_{6}} \lambda e^{-\lambda t_{4}} \lambda e^{-\lambda t_{6}} d t_{4} \\
& =2 \lambda\left(e^{-\lambda t_{6}}-e^{-2 \lambda t_{6}}\right)
\end{aligned}
$$

Let $t_{7}=t_{2}+t_{6}$. From (5),

$$
\begin{aligned}
f_{7}\left(t_{7}\right) & =\int_{t_{6}=0}^{t_{7}} 2 \lambda\left(e^{-\lambda t_{6}}-e^{-2 \lambda t_{6}}\right) \lambda e^{-\lambda\left(t_{7}-t_{6}\right)} d t_{6} \\
& =2 \lambda^{2}\left[\int_{t_{6}=0}^{t_{7}} e^{-\lambda t_{7}} d t_{6}-\int_{t_{6}=0}^{t_{7}} e^{-\lambda t_{7}} e^{-\lambda t_{6}} d t_{6}\right] \\
& =2 \lambda^{2} t_{7} e^{-\lambda t_{7}}-2 \lambda e^{-\lambda t_{7}}+2 \lambda e^{-2 \lambda t_{7}}
\end{aligned}
$$

Let $F_{7}\left(t_{7}\right)$ be the distribution function of $t_{7}$. Then $\bar{F}_{7}\left(t_{7}\right)=$ $1-F_{7}\left(t_{7}\right)$ is expressed as

$$
\begin{aligned}
\bar{F}_{7}\left(t_{7}\right) & =\int_{t=t_{7}}^{\infty} f_{7}(t) d t \\
& =2 \lambda t_{7} e^{-\lambda t_{7}}+e^{-2 \lambda t_{7}}
\end{aligned}
$$

From (6), probability $p_{2}$ is derived as

$$
\begin{aligned}
p_{2}= & \operatorname{Pr}\left[t_{7}>t_{1}+t_{3}\right] \\
= & \int_{t_{1}=0}^{\infty} \int_{t_{3}=0}^{\infty} \int_{t_{7}=t_{1}+t_{3}}^{\infty} f_{1}\left(t_{1}\right) f_{3}\left(t_{3}\right) f_{7}\left(t_{7}\right) d t_{7} d t_{3} d t_{1} \\
= & \int_{t_{1}=0}^{\infty} \int_{t_{3}=0}^{\infty} f_{1}\left(t_{1}\right) f_{3}\left(t_{3}\right) \bar{F}_{7}\left(t_{1}+t_{3}\right) d t_{3} d t_{1} \\
= & \int_{t_{1}=0}^{\infty} \int_{t_{3}=0}^{\infty} f_{1}\left(t_{1}\right) f_{3}\left(t_{3}\right)\left[2 \lambda t_{1} e^{-\lambda\left(t_{1}+t_{3}\right)}\right. \\
& \left.+2 \lambda t_{3} e^{-\lambda\left(t_{1}+t_{3}\right)}+e^{-2 \lambda\left(t_{1}+t_{3}\right)}\right] d t_{3} d t_{1} \\
= & A+B+C
\end{aligned}
$$

where

$$
\begin{aligned}
A & =\int_{t_{1}=0}^{\infty} \int_{t_{3}=0}^{\infty} f_{1}\left(t_{1}\right) f_{3}\left(t_{3}\right) 2 \lambda t_{1} e^{-\lambda\left(t_{1}+t_{3}\right)} d t_{3} d t_{1} \\
& =\int_{t_{1}=0}^{\infty} \int_{t_{3}=0}^{\infty} f_{1}\left(t_{1}\right) \lambda e^{-\lambda t_{3}} 2 \lambda t_{1} e^{-\lambda t_{1}} e^{-\lambda t_{3}} d t_{3} d t_{1} \\
& =\int_{t_{1}=0}^{\infty} 2 \lambda^{2} t_{1} f_{1}\left(t_{1}\right) e^{-\lambda t_{1}} \int_{t_{3}=0}^{\infty} e^{-2 \lambda t_{3}} d t_{3} d t_{1} \\
& =\int_{t_{1}=0}^{\infty} \lambda t_{1} f_{1}\left(t_{1}\right) e^{-\lambda t_{1}} d t_{1} \\
& =-\left.\lambda\left[\frac{d f_{1}^{*}(s)}{d s}\right]\right|_{s=\lambda} \\
B & =\int_{t_{1}=0}^{\infty} \int_{t_{3}=0}^{\infty} f_{1}\left(t_{1}\right) f_{3}\left(t_{3}\right) 2 \lambda t_{3} e^{-\lambda\left(t_{1}+t_{3}\right)} d t_{3} d t_{1} \\
& =\int_{t_{1}=0}^{\infty} 2 \lambda^{2} f_{1}\left(t_{1}\right) e^{-\lambda t_{1}} \int_{t_{3}=0}^{\infty} t_{3} e^{-2 \lambda t_{3}} d t_{3} d t_{1} \\
& =\int_{t_{1}=0}^{\infty} 2 \lambda^{2} f_{1}\left(t_{1}\right) e^{-\lambda t_{1}}\left(\frac{1}{2 \lambda}\right)^{2} d t_{1} \\
& =\frac{f_{1}^{*}(\lambda)}{2}
\end{aligned}
$$

and

$$
\begin{aligned}
C & =\int_{t_{1}=0}^{\infty} \int_{t_{3}=0}^{\infty} f_{1}\left(t_{1}\right) f_{3}\left(t_{3}\right) e^{-2 \lambda\left(t_{1}+t_{3}\right)} d t_{3} d t_{1} \\
& =\int_{t_{1}=0}^{\infty} \lambda f_{1}\left(t_{1}\right) e^{-2 \lambda t_{1}} \int_{t_{3}=0}^{\infty} e^{-3 \lambda t_{3}} d t_{3} d t_{1} \\
& =\left(\frac{1}{3}\right) \int_{t_{1}=0}^{\infty} f_{1}\left(t_{1}\right) e^{-2 \lambda t_{1}} d t_{1} \\
& =\frac{f_{1}^{*}(2 \lambda)}{3}
\end{aligned}
$$




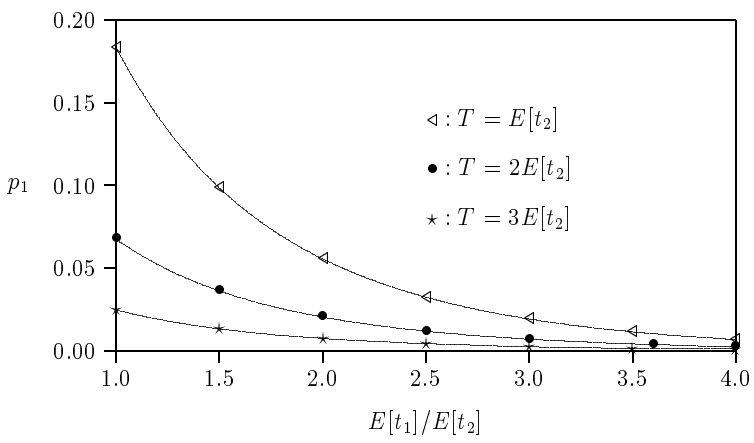

Fig. 3. Effects of $E\left[t_{1}\right] / E\left[t_{2}\right]$ and $T$ on $p_{1}\left(V_{1}=E\left[t_{1}\right]^{2}\right.$ and $\left.V_{2}=E\left[t_{2}\right]^{2}\right)$

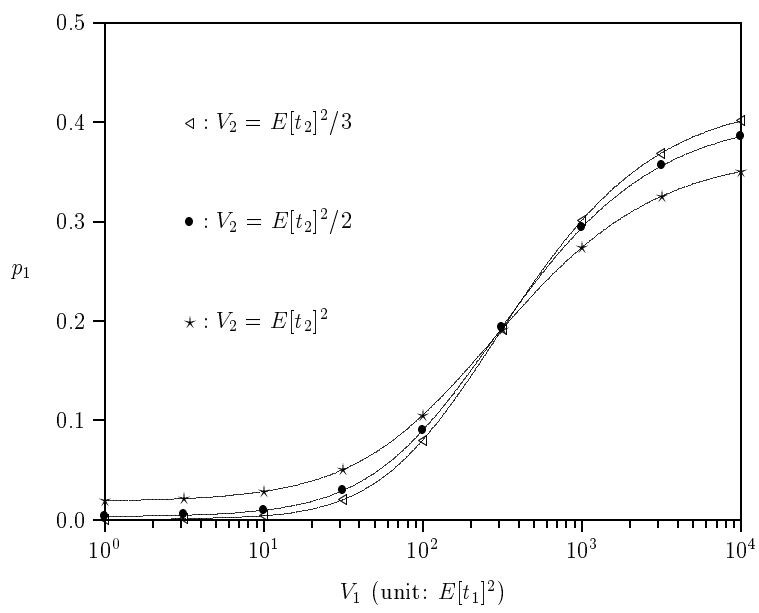

Fig. 4. Effects of $V_{1}$ and $V_{2}$ on $p_{1}\left(E\left[t_{1}\right]=3 E\left[t_{2}\right]\right.$ and $\left.T=E\left[t_{2}\right]\right)$

Substituting (8)-(10) into (7) to yield

$$
p_{2}=\frac{f_{1}^{*}(\lambda)}{2}+\frac{f_{1}^{*}(2 \lambda)}{3}-\left.\lambda\left[\frac{d f_{1}^{*}(s)}{d s}\right]\right|_{s=\lambda}
$$

If $t_{1}$ has the Gamma distribution, then

$$
p_{2}=\left(\frac{1}{2}\right)\left(\frac{\beta}{\beta+\lambda}\right)^{\alpha}+\left(\frac{1}{3}\right)\left(\frac{\beta}{\beta+2 \lambda}\right)^{\alpha}+\frac{\lambda \alpha \beta^{\alpha}}{(\beta+\lambda)^{\alpha+1}}
$$

Note that after ACM message transmission, the SS7 Answer Message (ANM) is sent from the called side to the calling side if the called party picks up the phone. The delay for local ANM transmission in our approach is $\max \left(t_{4}, t_{5}\right)$. The delay for international ANM transmission in the existing approach is $t_{1}+t_{3}$. The performance analysis for this signaling part directly follows the derivation for $p_{2}$ and the details are omitted.

\section{Performance Evaluation}

The analytic analysis in the previous section is validated against the simulation experiments. We utilize the Monte Carlo simulation to generate the delays, and then compare the lengths of these delays to produce the $p_{1}$ and $p_{2}$ values. In Figures 3-5, the $p_{1}$ and the $p_{2}$ curves are plotted based on the analytic analysis, and the symbols $\triangle$, $\bullet$, and $\star$ points are generated by simulation experiments. These figures indicate that our analytic and simulation results are consistent.

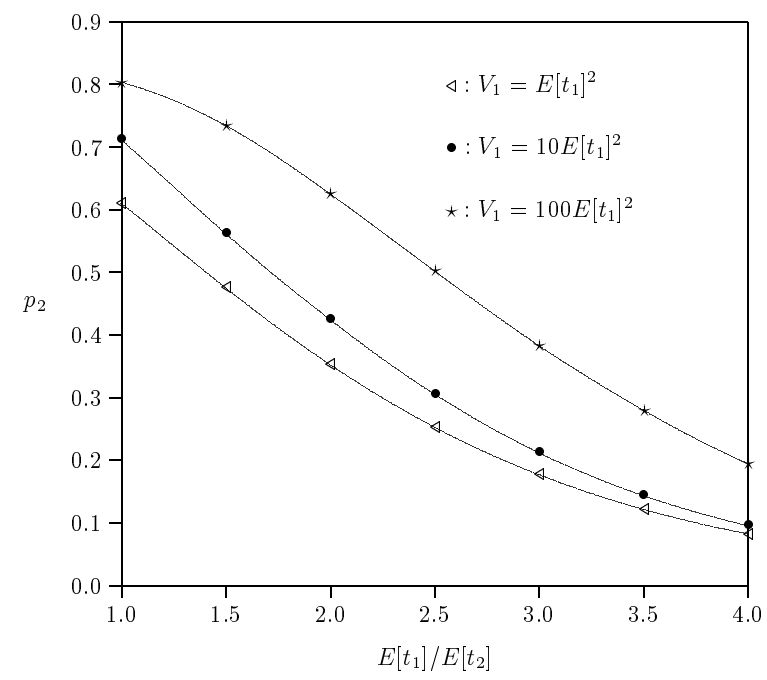

Fig. 5. Effects of $E\left[t_{1}\right] / E\left[t_{2}\right]$ and $V_{1}$ on $p_{2}\left(V_{2}=E\left[t_{2}\right]^{2}\right)$

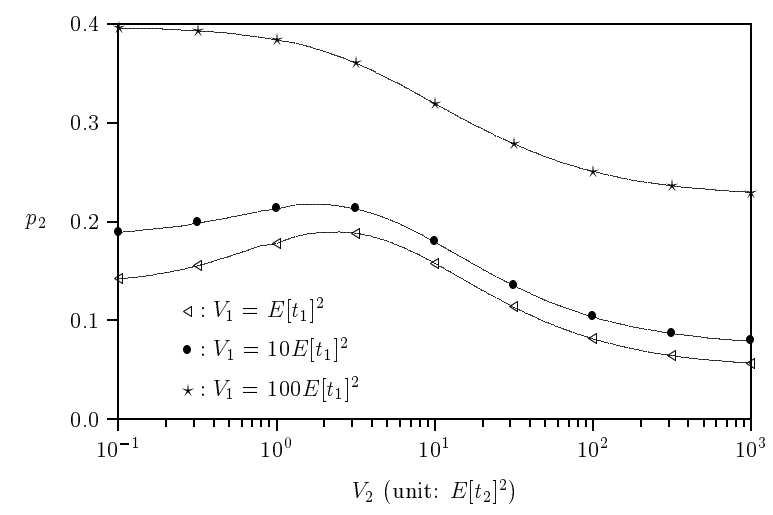

Fig. 6. Effects of $V_{1}$ and $V_{2}$ on $p_{2}\left(E\left[t_{1}\right]=3 E\left[t_{2}\right]\right)$

This section investigates the output measures $p_{1}$ and $p_{2}$. Figure 3 shows that $p_{1}$ decreases as $T$ or $E\left[t_{1}\right]$ increase. When $T \geq 2 E\left[t_{2}\right]$ and $E\left[t_{1}\right] \geq 2.5 E\left[t_{2}\right], p_{1}$ is smaller than $2.5 \%$.

Figure 4 shows that $p_{1}$ increases as $V_{1}$ increases. Large $V_{1}$ implies that there are more large $t_{1}$ intervals. Therefore, $p_{1}$ is an increasing function of $V_{1}$. In a telecom-grade network, the variances of transmission delays are restricted. In our example, it suffices to restrict $V_{1} \leq 10 E\left[t_{1}\right]^{2}$. When $V_{1}$ is small, $p_{1}$ increases as $V_{2}$ increases. Since $E\left[t_{2}\right]<E\left[t_{1}\right]$, when both $V_{1}$ and $V_{2}$ are small, it almost guarantees that $t_{2}<t_{1}$. Therefore, when $V_{1}$ is small, $p_{1}$ is an increasing function of $V_{2}$. When $V_{1}$ is large, $p_{1}$ increases as $V_{2}$ decreases. In this case, both long $t_{1}$ and $t_{2}$ intervals are likely to be observed when $V_{1}$ and $V_{2}$ increase. The variance effect on $t_{2}$ is more significant, and therefore $p_{1}$ is a decreasing function of $V_{2}$. Figure 5 shows that $p_{2}$ decreases as $E\left[t_{1}\right]$ increases or $V_{1}$ decreases. When $E\left[t_{1}\right] \geq 2.5 E\left[t_{2}\right]$, it is more likely that the call setup for our approach is faster than the existing approach.

Based on the simulation experiments, Figure 6 shows that for $V_{2} \leq E\left[t_{2}\right]^{2}, p_{2}$ is insignificantly affected by the change of $V_{2}$. When $V_{2} \geq 2 E\left[t_{2}\right]^{2}, p_{2}$ decreases as $V_{2}$ increases.

In typical international telecommunications networks, we anticipate that $E\left[t_{1}\right]>3 E\left[t_{2}\right]$. Therefore, Figures 3-6 indicate 
that the signaling performance of our approach is reasonably good. In our approach, $T$ is an important parameter, and the operator needs to determine an appropriate $T$ value. Our study indicates that $T>2 E\left[t_{2}\right]$ yields good performance in most cases we investigated.

\section{Conclusions}

This paper proposed a third-party solution that resolves the tromboning problem by replacing two international calls with two local calls. In our solution, local PSTN gateways are deployed in different countries. Several issues are discussed below.

Issue 1: The solution provider is typically a VoIP service provider so that call signaling can be delivered through the IP network (see Figure $2(\mathrm{k})$ ). The solution provider needs not be a mobile service provider. In other words, the roaming customer can select arbitrary mobile service operator at her home country. The mobile phone number of a customer in our approach is "hidden", and we do not anticipate that this number is directly dialed. In fact, this number is not supposed to be distributed to other people, and needs not to be even known by the customer. Therefore, it would be better that this mobile phone number is internally allocated by the solution provider.

Issue 2: The solution provider may only deploy the PSTN gateways in some countries based on its business strategy. Following the local telecommunications regulation, a block of E.164 phone numbers (e.g., the block including 4433333333 in our example) are assigned to the PSTN gateway in a country if the solution provider is also a telecom operator in that country. In this case, the PSTN gateway is assigned an SS7 number known by the originating switch (Figure 2 (b)). When someone dials the local phone number of the roaming customer, the originating switch routes the call to the PSTN gateway using the gateway's SS7 number. Alternatively, the solution provider can be an enterprise customer of a local telecom operator that allocates several dedicated lines to the PSTN gateway. In this case, the local telecom operator provides a block of phone numbers (including 4433333333 in our example) to the solution provider. The range of the phone numbers can be much larger than the dedicated lines leased to the solution provider.

Issue 3: The MS of a roaming customer can be a standard smart phone with an application-level software installed by the solution provider, which does not modify the standard mobile phone software in the MS. This applicationlevel software maintains a PSTN gateway address table. When the roaming customer enters the international roaming mode, she is asked to type the visited country name or enters the country code of the visited country (e.g., code 1 for the US, code 86 for China, and so on). The MS then uses the country code to identify the address of the PSTN gateway in the table. This address is used to deliver the IAM message at Step B.4 (b).

In this paper, we also conducted analytic modeling and simulation experiments to show that our approach is effective in call setup signaling. To conclude, our solution resolves the tromboning problem of international mobile call setup. Another advantage of our solution is that we only need to deploy local PSTN gateways without modifying the existing mobile telecommunications systems. Furthermore, our approach can be easily extended to accommodate the scenarios when both the called and the calling parties are in different countries. The details are treated in a separate paper. As a final remark, our approach is pending patents of the US, China, Japan, and Taiwan.

\section{ACKNOWLEDGMENT}

The author would like to thank M.-H. Tsai, Y.-C. Sung, H.-W. Dai, and H.-Y. Lee for their efforts in checking the analytic derivation and simulation validation. This work was sponsored in part by NSC 96-2752-E-009-005-PAE, NSC 962219-E-009-019, NSC 96-2221-E-009-020, Intel, Chung Hwa Telecom, IIS/Academia Sinica, ITRI/NCTU Joint Research Center and MoE ATU.

\section{REFERENCES}

[1] B. Li, et al., "Call admission control for voice/data integrated cellular networks: performance analysis and comparative study," IEEE J. Select. Areas Commun., vol. 22, no. 4, pp. 706-718, 2004.

[2] Y.-B. Lin, and I. Chlamtac, Wireless and Mobile Network Architectures. John Wiley \& Sons, 2001.

[3] 3GPP, "GPRS Service Descripton; Stage 2," 2007, 3G TS 23.060 (Version 7.4.0).

[4] Y.-J. Cho, Y.-B. Lin, and C.-H. Rao, "Reducing the Network cost of call delivery to GSM roamers," IEEE Network Mag., vol. 11, no. 5, pp. 19-25, Sept./Oct. 1997.

[5] Y.-B. Lin and A.-C. Pang, Wireless and Mobile All-IP Networks. John Wiley \& Sons, 2005.

[6] Y. Fang, "Modeling and performance analysis for wireless mobile networks: a new analytical approach," IEEE/ACM Trans. Networking, vol. 13, no. 5, pp. 989-1002, 2005.

[7] - "General modeling and performance analysis for location management in wireless mobile networks," IEEE Trans. Computers, vol. 51, no. 10, pp. 1169-1181, 2002.

[8] Y.-B. Lin, P.-J. Lee, and I. Chlamtac, "Dynamic periodic location area update in mobile networks," IEEE Trans. Veh. Technol., vol. 51, no. 6, pp. 1494-1501, 2002.

[9] H.-N. Hung, Y.-B. Lin, N.-F. Peng, and S.-I. Sou, "Connection failure detection mechanism of UMTS charging protocol," IEEE Trans. Wireless Commun., vol. 5, no. 5, pp. 1180-1186, 2006.

[10] P. Lin and G.-H. Tu, "An improved GGSN failure restoration mechanism for UMTS,” ACM Wireless Networks, vol. 12, no. 1, pp. 91-103, 2006.

[11] C.-H. Gan, N.-C. Perng, P. Lin, T.-W. Kuo, "Time division based cyclic scheduling for UMTS high-speed downlink shared-channels," IEEE Trans. Veh. Technol., vol. 56, no. 4, pp. 2086-2094, 2007.

[12] S.-R. Yang, "Dynamic power saving mechanism for 3G UMTS system," ACM/Springer Mobile Networks and Applications (MONET), 2006, published online.

[13] F. P. Kelly, Reversibility and Stochastic Networks. John Wiley \& Sons, 1979.

[14] E. D. Lazowska, J. Zahorjan, G. S. Graham, and K. C. Sevcik, Quantitative System Performance. Prentice Hall, 1984.

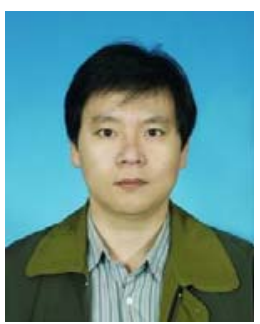

Yi-Bing Lin (M'96-SM'96-F'03) is Chair Professor and Dean of College of Computer Science, National Chiao Tung University. His current research interests include mobile computing and cellular telecommunications services. Dr. Lin has published over 200 journal articles and more than 200 conference papers. He is the co-author of the books Wireless and Mobile Network Architecture (with Imrich Chlamtac; published by Wiley, 2001) and Wireless and Mobile All-IP Networks (with Ai-Chun Pang; published by Wiley, 2005). Dr. Lin is an IEEE Fellow, ACM Fellow, AAAS Fellow, and IEE Fellow. 